\title{
Payload Mass Identification of a Single-Link Flexible Arm Moving under Gravity: An Algebraic Identification Approach
}

\author{
Juan Carlos Cambera, Andres San-Millan, and Vicente Feliu-Batlle
}

Universidad de Castilla-La Mancha, 13071 Ciudad Real, Spain

Correspondence should be addressed to Vicente Feliu-Batlle; vicente.feliu@uclm.es

Received 8 December 2014; Accepted 27 May 2015

Academic Editor: Reza Jazar

Copyright ( 2015 Juan Carlos Cambera et al. This is an open access article distributed under the Creative Commons Attribution License, which permits unrestricted use, distribution, and reproduction in any medium, provided the original work is properly cited.

We deal with the online identification of the payload mass carried by a single-link flexible arm that moves on a vertical plane and therefore is affected by the gravity force. Specifically, we follow a frequency domain design methodology to develop an algebraic identifier. This identifier is capable of achieving robust and efficient mass estimates even in the presence of sensor noise. In order to highlight its performance, the proposed estimator is experimentally tested and compared with other classical methods in several situations that resemble the most typical operation of a manipulator.

\section{Introduction}

Flexible link robotics is a research field focused on building robots with better performance than the conventional robots. The flexibility of these robots is a consequence of using links with lower sectional area and lighter materials than its rigid counterpart. Higher operational speed, lower energy consumption, better transportability, and lower cost are only a few advantages of the flexible link robots over the traditional rigid manipulators. These advantages can only be obtained by facing very challenging problems on modelling and control that after four decades have not been completely solved.

The flexible link robots are systems characterised by nonlinear ordinary, coupled, and partial differential equations, whose exact solution is not viable practically. This had led to look for models with manageable complexity but still reliable and useful for the design of controllers. From the control perspective, the same characteristics that improve the performance of these robots have led to vibration problems that undermine the positioning of the end effector. The solution to these problems can be very difficult considering the complexity of the model and, in the most general case, its nonminimum phase nature. Surveys dealing with dynamic modelling and control of flexible link robots can be found in [1-3].
In flexible link robotics, to guarantee an accurate positioning in pick and place tasks is a very important problem to solve. One of the main obstacles to overcome is to design a control algorithm capable of cancelling the vibrations when the dynamics is affected by changes in the payload mass. When these changes are not considered in the control design, the algorithm may lose accuracy and effectiveness in the vibration suppression and, in some cases, may become unstable.

Several works have addressed the problem from the adaptive control point of view. Most of them rely on the indirect methods category, which consists of two clearly differentiated stages (there are some early research works that use a direct approach; Siciliano et al. [4] and Yuh [5] applied the Model Reference Adaptive Control (MRAC)). In the first stage, an online identification of the system parameters is needed. In the second one, the parameters identified in the first stage are used to adjust the adaptive control law, in such a way that the overall performance of the system is improved. This paper is devoted to the real time characterization of the parameter that is most likely to change in a robot: the payload. In particular, we want to identify the tip mass, as we assume that the payload polar moment of inertia is negligible. Once this parameter has been identified, to update the dynamic model 
of the arm is immediate, and to recompute the controller parameters is straightforward.

A payload change affects in two ways a flexible link robot, it changes the vibration frequencies of the links, and it changes the motor torques demanded for a specific maneuver. Hence the identification algorithms can be classed, on a similar way, into frequency based approaches and model based approaches.

The frequency based approaches, normally, do not depend explicitly on the robot's model but on the output where the vibrations appear and in some cases on the input. The more classical approaches are normally based on the FFT [6]. The adaptive notch filter [7] is one of the preferred methods because of its fast convergence rates and its low computational burden. Other approaches, like [8], have considered adaptive observers to perform simultaneously frequency and states estimation. A more recent work uses algebraic identification to estimate amplitude, frequency, and phase of a sinusoidal signal in the presence of noise and DC-offsets [9], which are two common problems not explicitly considered in the methods aforementioned.

In the second category, which we referred to as model based approaches, the payload mass is identified by using the dynamic model of the robot and the input and output signals. Least square based techniques, like [10-12], cover most of the work carried out under this category, but there are some other alternatives based on algebraic manipulations of the model transfer functions [13], Kalman filtering [14], or the already mentioned algebraic identification technique [15] that are also worth mentioning.

From the vibration control point of view, the frequency based approaches are at a disadvantage. The frequency based approaches require the system to vibrate at least a cycle fraction before the identification can be carried out. This condition goes against the main goal of the control algorithm, the vibration suppression, where a very fast identification is required. In order to update the controller as soon as possible during the trajectory execution, on the other hand, the model based approaches have proved to be highly reliable in problems concerning dynamic linear systems, but its applicability to nonlinear systems is not straightforward and may imply in some cases numerical differentiation of noisy signals.

In this paper, we will focus on the problem of real time identification of the tip mass of a single-link flexible arm that moves in a vertical plane under the effects of the gravity. The algorithm follows a model based approach and it is based on the algebraic identification framework, proposed in [16], and it generalizes a previous research work presented in [15]. Unlike the previous research work, where only movements in the horizontal plane were considered and then a linear model was used, in this paper we deal with a nonlinear dynamic model as a consequence of taking the gravity into account. This leads to a more complex problem, where most of the real time identification techniques developed up to date cannot be applied. Preliminary results of this identification algorithm were presented in [17]. This paper details our new identification algorithm, proposes several improvements, and presents a comparative analysis with other identification methods.

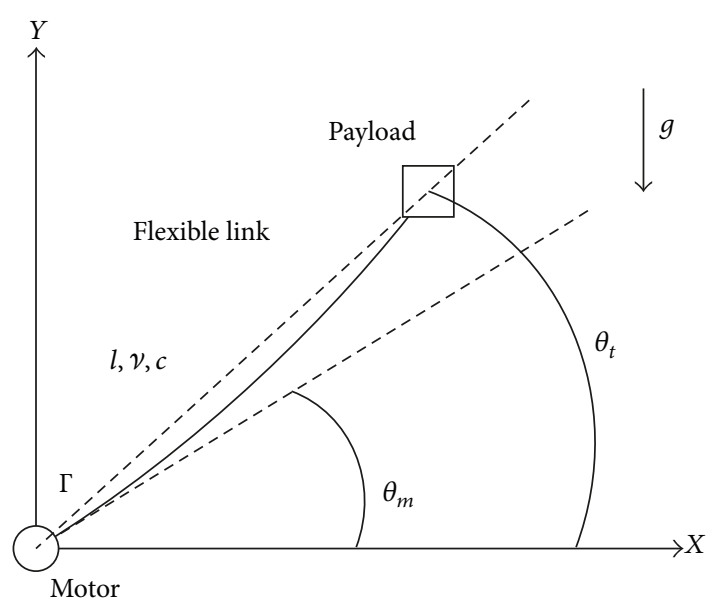

FIGURE 1: Robotic system scheme.

The algebraic identification provides a very fast and simple solution for online parameter estimation in systems where the parameters are piecewise constant (change from one constant value to another unpredictably). This methodology is fundamentally different from other approaches in some basics aspects: (a) it does not require any statistical knowledge of the noise corrupting the signals; therefore, classical Gaussian noise assumptions are not necessary; (b) it does not need to compute iterative time derivatives of noise corrupted signals; (c) it is not an asymptotic approach; and (d) it does not require persistently exciting inputs in order to make the system identifiable [18].

This paper is organised as follows. Section 2 presents the dynamic model of the flexible-link robot. In Section 3, the design of the algebraic identification algorithm is presented. Section 4 is devoted to the analysis and comparison of the experimental results. Conclusions and future work are presented in Section 5.

\section{Dynamic Model}

Figure 1 shows a schematic representation of the flexible link robot. It consists of a motor and a flexible beam that bends on the vertical plane and therefore is affected by the gravitational force. One end of the beam is clamped to the shaft of the motor, while the other end moves freely and carries a payload. The model we introduce in this section was prepared according to the lumped mass method presented in [19] and relies on the following assumptions.

(i) The link mass is negligible in comparison to the tip mass.

(ii) The payload is considered as a point mass; therefore, its polar moment of inertia can be neglected.

(iii) The deflections are elastic and small in relation to the link's length, so that geometrical linearity can be assumed.

(iv) Torsion and compression effects of the link are small in relation to the deflections. 
The dynamic model relates the coupling torque between the motor and the link $(\Gamma)$ to the angular motor position $\left(\theta_{m}\right)$ and the angular tip position $\left(\theta_{t}\right)$. The dynamic model of the system is given by

$$
\begin{aligned}
& \Gamma(t)=m l^{2} \ddot{\theta}_{t}(t)+\nu \dot{\theta}_{t}(t)+m g l \cos \left(\theta_{t}(t)\right), \\
& \Gamma(t)=c\left(\theta_{m}(t)-\theta_{t}(t)\right),
\end{aligned}
$$

where $m$ is the payload mass, $l$ is the link's length, $v$ is the viscous friction damping coefficient, and $c$ is a stiffness constant that can be defined in function of Young's modulus $(E)$ and the cross-sectional moment of inertia $(I)$ as $c=3 E I / l$. This last expression is a result of applying the lumped mass methodology developed in [19] to a single-link flexible robot that considers the assumptions presented above.

For the algebraic estimation algorithm that we develop in this paper, the torque measured at the base of the link, the angular motor position, and the angular tip position need to be known. The sensory system of our robot provides the angle of the motor $\left(\theta_{m}\right)$ and the coupling torque $(\Gamma)$ at the base of the link. The angular tip position $\left(\theta_{t}\right)$ is not measured but it can be estimated as follows:

$$
\theta_{t}^{e}(t)=\theta_{m}(t)-\frac{\Gamma(t)}{c} .
$$

Here it is important to notice that the estimation of tip position, $\theta_{t}^{e}$, is completely independent from the payload mass $m$, whose value is in principle unknown.

\section{Algebraic Identification Algorithm}

In this section the algebraic methodology, presented in [16], is used to design an online algorithm to identify the payload mass of a flexible link robot moving under gravity. The basic methodology works from the premise that the parameters are constant throughout time, but it can be extended to identification problems where the parameters are piecewise constant; that is, payload changes in a pick and place task. For the sake of clarity, the design methodology is explained in three stages. The design procedure considers (1) and (3). The presence of the cosine function in (1) determines its nonlinear characteristic in the tip position variable $\left(\theta_{t}\right)$. From the identification point of view, however, and considering that the tip position can be estimated from (3), this nonlinear equation can be considered linearly identifiable in its parameters $m$ and $\nu$. Taking this into consideration the design methodology is as follows.

3.1. First Stage: Algebraic Reformulation of the Problem. Performing an online estimation of the parameters in (1) implies, for some identification techniques, numerical differentiations of a noise corrupted signal $\theta_{t}^{e}$. As it is well known, these operations amplify the noise in the resulting signals and affect adversely the performance of the identification algorithms. The algebraic identification framework provides a methodology to avoid this issue. In this section, we formulate a new mathematical relationship between the measured signals and the parameters of the model by applying standard operational calculus and algebraic operations. This new expression, compared with the original model, is purposefully formulated to be independent from time derivatives and initial conditions. The procedure is as follows.

First, let us apply the Laplace transform to (1):

$$
\begin{aligned}
\Gamma(s)= & m l^{2}\left[s^{2} \theta_{t}(s)-s \theta_{t}\left(t_{0}\right)-\dot{\theta}_{t}\left(t_{0}\right)\right] \\
& +v\left[s \theta_{t}(s)-\theta_{t}\left(t_{0}\right)\right]+\operatorname{mgl\chi }(s),
\end{aligned}
$$

where $\theta_{t}\left(t_{0}\right)$ and $\dot{\theta}_{t}\left(t_{0}\right)$ represent unknown initial conditions and $\chi(s)$ is the Laplace transform of the signal $\cos \left(\theta_{t}(t)\right)$. Taking two times derivatives with respect to $s$, the initial conditions are eliminated:

$$
\begin{aligned}
\frac{d^{2} \Gamma(s)}{d s^{2}}= & m l^{2}\left[2 \theta_{t}(s)+4 s \frac{d \theta_{t}(s)}{d s}+s^{2} \frac{d^{2} \theta_{t}(s)}{d s^{2}}\right] \\
& +v\left[2 \frac{d \theta_{t}(s)}{d s}+s \frac{d^{2} \theta_{t}(s)}{d s^{2}}\right]+m g l \frac{d^{2} \chi(s)}{d s^{2}} .
\end{aligned}
$$

In order to eliminate the derivatives in the time domain (positive power of $s$ ), we multiple both sides by $s^{-2}$ to obtain

$$
\begin{aligned}
s^{-2} \frac{d^{2} \Gamma(s)}{d s^{2}}= & m l^{2}\left[2 s^{-2} \theta_{t}(s)+4 s^{-1} \frac{d \theta_{t}(s)}{d s}+\frac{d^{2} \theta_{t}(s)}{d s^{2}}\right] \\
& +v\left[2 s^{-2} \frac{d \theta_{t}(s)}{d s}+s^{-1} \frac{d^{2} \theta_{t}(s)}{d s^{2}}\right] \\
& +m g l s^{-2} \frac{d^{2} \chi(s)}{d s^{2}}
\end{aligned}
$$

Equation (6) can be written in the time domain by applying the inverse Laplace transform (there are few properties that are specially useful for this problem; if we denote the inverse Laplace transform by $\mathscr{L}^{-1}$, then it is well known that $\mathscr{L}^{-1} s(\cdot)=d / d t(\cdot), \mathscr{L}^{-1} 1 / s(\cdot)=\int_{0}^{t}(\cdot)(\sigma) d \sigma$, and $\left.\mathscr{L}^{-1} d^{v} / d s^{v}(\cdot)=(-1)^{v} t^{v}(\cdot)\right)$. The resulting equation is given by (we denote by $\left(\int^{(j)} \phi(t)\right)$ the integral expression $\int_{0}^{t} \int_{0}^{\sigma_{1}} \cdots$ $\int_{0}^{\sigma_{j-1}} \phi\left(\sigma_{j}\right) d \sigma_{j} \cdots d \sigma_{1}$ with the definition $\left(\int \phi(t)\right)=$ $\left.\int_{0}^{t} \phi\left(\sigma_{1}\right) d \sigma_{1}\right)$

$$
\begin{aligned}
\int^{(2)} t^{2} \Gamma(t)= & m l^{2}\left[2 \int^{(2)} \theta_{t}(t)-4 \int t \theta_{t}(t)+t^{2} \theta_{t}(t)\right] \\
& +v\left[-2 \int^{(2)} t \theta_{t}(t)+\int t^{2} \theta_{t}(t)\right] \\
& +m g l \int^{(2)} t^{2} \cos \left(\theta_{t}(t)\right)
\end{aligned}
$$

and in a more compact form as

$$
q(t)=m\left[l^{2} \beta(t)+g l \xi(t)\right]+\nu \eta(t),
$$


where

$$
\begin{aligned}
& q(t)=\int^{(2)} t^{2} \Gamma(t), \\
& \beta(t)=2 \int^{(2)} \theta_{t}(t)-4 \int t \theta_{t}(t)+t^{2} \theta_{t}(t), \\
& \xi(t)=\int^{(2)} t^{2} \cos \left(\theta_{t}(t)\right), \\
& \eta(t)=-2 \int^{(2)} t \theta_{t}(t)+\int t^{2} \theta_{t}(t) .
\end{aligned}
$$

Functions $q(t), \beta(t), \xi(t)$, and $\eta(t)$ can be expressed as the outputs of the following time-varying linear and unstable filters in perturbed Brunovsky's canonical form:

$$
\begin{aligned}
q(t) & =z_{1}, \\
\dot{z}_{1} & =z_{2}, \\
\dot{z}_{2} & =t^{2} \Gamma(t), \\
\beta(t) & =z_{3}+t^{2} \theta_{t}(t), \\
\dot{z}_{3} & =z_{4}-4 t \theta_{t}(t), \\
\dot{z}_{4} & =2 \theta_{\mathrm{t}}(t), \\
\xi(t) & =z_{5}, \\
\dot{z}_{5} & =z_{6}, \\
\dot{z}_{6} & =t^{2} \cos \left(\theta_{t}(t)\right), \\
\eta(t) & =z_{7}, \\
\dot{z}_{7} & =z_{8}+t^{2} \theta_{t}(t), \\
\dot{z}_{8} & =-2 t \theta_{t}(t) .
\end{aligned}
$$

The implementation of this algorithm requires as inputs the coupling torque measurements $(\Gamma)$ and the estimation of the tip position $\left(\theta_{t}^{e}\right)$, presented in (3). Notice that even though the original expression (1) and the resulting equation (8) have the same structure, the latter does not depend on time numerical differentiations.

3.2. Second Stage: Parameter Calculation. To identify the parameters of a system we need at least the same number of linearly independent equations as unknown parameter. The algebraic identification exploits the advantages of the resulting expression for the algebraic reformulation of the problem, expression (8) in our case, to define these independent equations. In this context, several approaches have been proposed, not only to define these equations, but also to enhance the performance of the identifier in the presence of noise in the measurements. In the following paragraphs, first, we briefly describe the most relevant approaches, and then we detail the procedure followed in this paper. The approaches we mention are not necessarily referring to our specific application, but they could be easily adapted to our problem.

A first approach is to generate as many linearly independent equations as parameters have to be estimated by successive differentiation of (8), which was obtained after algebraic manipulations. This approach was developed in [20] in the context of multiple harmonic signals identification. Note that (8) is a single equation which involves two parameters to be estimated. Then we need an extra equation for the identification. Once the resulting system of equations is solved, and in those cases where high frequency noise is present in the measurements, a low-pass filtering has to be carried out in order to improve the signal to noise ratio of the parameter estimates. Depending on the nature of the measurements considered, the equations defined in this approach might not be completely linearly independent all the time. When this happens, a local loss of identifiability may occur. In [21], this drawback was solved by using invariant nonlinear filtering.

Other approaches to define this system of equations are to use the equation resulting from the algebraic reformulation of problem, (8) in our case, and to evaluate it at different times. Under this approach, an overdetermined system of equations is defined by taking a large number of points in time. This action allows us to achieve the two goals mentioned above at the same time: to identify the parameters and to reduce the effects of the noise present in the measurements. The bigger the number of evaluation instants is, the greater the lowpass filtering effect is. To solve this overdetermined system of equations a least squares fitting based method is normally considered. In [22], the least squares method in connection with algebraic identification was proposed to identify the parameters for induction motors. In order to increase the computational efficiency of this stage, in [23], a continuous least squares approximation was implemented in a recursive way to identify the two main vibration modes of a flexible structure. In [24] the adjustment of the overdetermined system was carried out by using a recursive least squares algorithm. This last identifier was designed to determine the parameters of a servo model. In the present paper we use the continuous least square approximation with a recursive implementation of the involved integrals. A detailed description is presented below.

The goal of the following steps is to determine the unknown parameters $m$ and $\nu$ from (8). However, at the end of this section we will only center our attention on the value of the parameter $m$, while the value of the parameter $v$ will be discarded. The parameter $v$, associated with the viscous friction, was introduced in the model only to improve the behaviour of the estimator. According to our observations, an approximate model of the damping enhances the performance in terms of precision. Having made this clarification, we define the following cost function:

$$
\varepsilon=\int_{0}^{t}\left\{\left[l^{2} \beta(\tau)+g l \xi(t) \eta(\tau)\right] \cdot\left[\begin{array}{c}
m \\
v
\end{array}\right]-q(\tau)\right\}^{2} d \tau,
$$


and its minimization leads to

$$
\begin{gathered}
{\left[\begin{array}{l}
m \\
v
\end{array}\right]=\left[\int_{0}^{t}\left[\begin{array}{c}
l^{2} \beta(\tau)+g l \xi(\tau) \\
\eta(\tau)
\end{array}\right]\right.} \\
\left..\left[\begin{array}{c}
l^{2} \beta(\tau)+g l \xi(\tau) \\
\eta(\tau)
\end{array}\right]^{T} d \tau\right]^{-1} \\
\cdot \int_{0}^{t}\left[\begin{array}{c}
l^{2} \beta(\tau)+g l \xi(\tau) \\
\eta(\tau)
\end{array}\right] q(\tau) d \tau .
\end{gathered}
$$

This calculation can be efficiently implemented in a recursive way as follows. Let us rewrite (12) as

$$
\left[\begin{array}{l}
m \\
v
\end{array}\right]=A^{-1}(t) B(t)=\left[\begin{array}{ll}
a_{11}(t) & a_{12}(t) \\
a_{21}(t) & a_{22}(t)
\end{array}\right]^{-1}\left[\begin{array}{l}
b_{1}(t) \\
b_{2}(t)
\end{array}\right],
$$

where

$$
\begin{aligned}
a_{11}(t) & =\int_{0}^{t}\left(l^{2} \beta(\tau)+g l \xi(\tau)\right)^{2} d(\tau), \\
a_{12}(t), a_{21}(t) & =\int_{0}^{t}\left(l^{2} \beta(\tau)+g l \xi(\tau)\right)(\eta(\tau)) d(\tau), \\
a_{22}(t) & =\int_{0}^{t}\left(\eta(\tau)^{2}\right) d(\tau), \\
b_{1}(t) & =\int_{0}^{t}\left(l^{2} \beta(\tau)+g l \xi(\tau)\right) q(\tau) d(\tau), \\
b_{2}(t) & =\int_{0}^{t} \eta(\tau) q(\tau) d(\tau) .
\end{aligned}
$$

Assume that functions $\beta(t), \xi(t), \eta(t)$, and $q(t)$ are sampled at discrete times $t=k T_{s}, k=1,2,3, \ldots$, where $T_{s}$ is the sampling time. If we define the function $\phi^{T}[k]=$ $\left[\left(l^{2} \beta[k]+g l \xi[k]\right) \eta[k]\right]$, the matrices $A(t)$ and $B(t)$ can be computed recursively in discrete time as follows:

$$
\begin{aligned}
& A[k]=A[k-1]+\phi[k] \phi^{T}[k] T_{s}, \\
& B[k]=B[k-1]+\phi[k] q[k] T_{s}, \\
& A[0]=0_{[2 \times 2]}, \\
& B[0]=0_{[2 \times 1]},
\end{aligned}
$$

where $0_{[2 \times 2]}$ and $0_{[2 \times 1]}$ are zero matrices and their subscripts represent their dimensions. The parameters $m$ and $v$ can be computed from these matrices as

$$
\left[\begin{array}{c}
m[k] \\
v[k]
\end{array}\right]=A^{-1}[k] B[k] .
$$

3.3. Third Stage: Resetting and Switching-Off Considerations. In the algorithm presented above, we considered that the uncertain parameters remain constant throughout the time.
However, this condition is not wide enough to solve the payload identification problem of the typical pick and place tasks. In this application, the uncertain parameter $m$ may suddenly change to a new constant value as the manipulator takes or drops an object. To detect payload changes, in this case, we require the algorithm to be reinitiated once it has achieved an estimate of the current parameters. But the instant when the parameter is accurately computed is, in principle, unknown and method to determine it is therefore needed.

To detect when the identification algorithm has converged, we consider in this paper the strategy proposed in [25]. In this strategy, the convergence time is expressed in terms of the moving average and the moving standard deviation of the parameter to identify. In our case, if we define $m[k]$ as the payload mass estimate at the sample $k$, its moving average $(E[m[k]])$ and its moving standard deviation $(\sigma[m[k]])$ are defined as follows:

$$
\begin{aligned}
E[m[k]] & =\frac{1}{M} \sum_{i=0}^{M-1} m[k-i], \\
\sigma[m[k]] & =\sqrt{\frac{1}{M} \sum_{i=0}^{M-1}(m[k-i]-E[m[k]])^{2}},
\end{aligned}
$$

where the length of the window $(M)$ defines the set of samples considered in the calculations. The payload mass estimated $m[k]$ is said to have converged when the following criterium is fulfilled:

$$
\frac{\sigma[m[k]]}{|E[m[k]]|} \leq \Delta .
$$

The constant $\Delta$ is the tolerance parameter and should be set up according to the application's requirements. If the application needs a high precision, a small $\Delta$ should be provided. On the other hand, if the application requires a fast estimation, a greater $\Delta$ should be considered.

There are some other alternatives to the procedure presented above. In [21] the convergence time was computed in terms of an artificial parameter called the sentinel parameter. This parameter emulates, in terms of time of convergence, the behaviour of the remaining system parameters, but, unlike the others, its value converges to an arbitrary value explicitly defined beforehand.

On a final note, we would like to point that for those applications where the parameters never change, and only a unique estimate of the parameters is necessary, it is advisable to switch off the algorithm once it has converged. The unstable nature of the filters involved in the identification may produce an arithmetic overflow when long time has passed. Given the fast nature of the algebraic identification, the algorithm is expected to be reset long before any numerical problem might arise.

\section{Experiments}

In this section, the algebraic identification algorithm presented above is experimentally validated and compared against least squares fitting based approaches. 


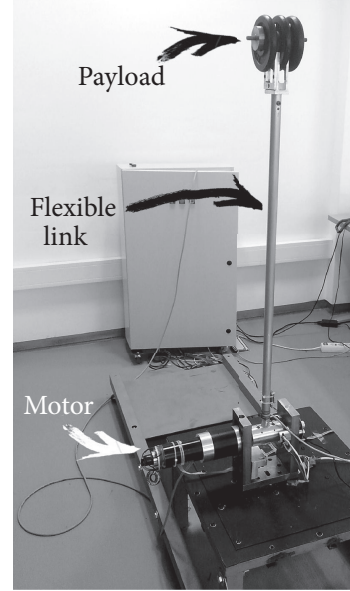

FIGURE 2: Flexible single-link robot platform.

4.1. Experimental Platform. Figure 2 shows the single-link flexible robot considered in the experiments. It is composed of a Maxon DC motor with gear reduction, a tubular duraluminium link, and a mass-adjustable payload structure. The link is connected at one side to the gear shaft, while at the same time holding the payload structure at its free end. The motor position commands are sent from a National Instrument PXI real time system and are executed by a Maxon EPOS motor driver. The control loops are executed with a sampling time of $T_{s}=2$ mseconds. The sensory system consists of strain gages placed at the base of the link and two incremental encoders to measure the motor position and gear shaft position. The strain measurements at the base of the link are used to estimate the coupling torque $(\Gamma)$, while the outer encoder is used to measure the exact orientation of the link at its base $\left(\theta_{m}\right)$. The inner encoder measurements were not taken into account because the backlash in the gear reduction would produce erroneous estimates of the position of the base of the link. However, the outer encoder has a relatively low resolution of 1024 pulses/revolution. Under these conditions, the measured signals used by the identification algorithm, $\left(\theta_{m}\right)$ and $(\Gamma)$, are very noisy, and their time derivatives, as might be expected, are even noisier and should not be used for an identification algorithm. To illustrate this, Figure 3 shows, for an arbitrary experiment, the signals relevant for the identification algorithm: the estimation of the tip position $\left(\theta_{t}^{e}\right)$, computed as it was shown in (3), from the experimental records of $\theta_{m}$ and $\Gamma$; the first- and secondorder time numerical differentiation of such estimation of the tip position, $\left(\dot{\theta}_{t}^{e}\right)$ and $\left(\ddot{\theta}_{t}^{e}\right)$; and the coupling torque $(\Gamma)$. As a consequence of the limited resolution of the outer encoder, the estimated signals $\left(\dot{\theta}_{t}^{e}\right)$ and $\left(\ddot{\theta}_{t}^{e}\right)$ present important discontinuities as shown in Figures 3(b) and 3(c). These two differentiated signals are relevant for the other identification algorithms that we will use as references, in the following sections, to evaluate our algebraic identification algorithm.

The most relevant features of the robot are described in Table 1 . The stiffness constant $c$ and damping coefficient
TABLE 1: Flexible robot parameters.

\begin{tabular}{lccc}
\hline Parameter & Units & Symbol & Value \\
\hline Beam length & $(\mathrm{m})$ & $l$ & 1.045 \\
$\begin{array}{l}\text { Beam rotational stiffness } \\
\text { Beam viscous damping }\end{array}$ & $\left(\mathrm{N} \cdot \mathrm{m} \cdot \mathrm{rad}^{-1}\right)$ & $c$ & 2498.2 \\
coefficient & $\left(\mathrm{N} \cdot \mathrm{m} \cdot \mathrm{s} \cdot \mathrm{rad}^{-1}\right)$ & $v$ & 0.2 \\
Payload mass 1 & $(\mathrm{Kg})$ & $m_{1}$ & 1.150 \\
Payload mass 2 & $(\mathrm{Kg})$ & $m_{2}$ & 1.690 \\
Payload mass 3 & $(\mathrm{Kg})$ & $m_{3}$ & 2.930 \\
\hline
\end{tabular}

$v$ were experimentally identified. A frequency based identification, explained in [26], was considered to adjust the parameter $c$. The damping coefficient $\nu$ provided in this table is given for indicative purpose only, and it is not required for the identification algorithm. This parameter was computed by adjusting approximately the decay rate of the residual vibrations in the time domain for a specific experiment, but it might change considerably depending on the experiment.

4.2. Definition of the Experiments. To validate the algebraic identifier, three types of experiments were defined to reproduce the typical operations of a manipulator. These experiments consider three payload masses $\left(m_{1}, m_{2}, m_{3}\right)$, whose values are given in Table 1 . The motor position was controlled using the algebraic controller explained in [26], while no tip vibration control algorithm was considered. It is noteworthy that the algebraic identifier presented does not depend on the control algorithm used for the motor position. In fact, it only considers the dynamics of the flexible link.

4.2.1. Experiment Type 1. The motor position $\left(\theta_{m}\right)$ tracks a fourth-order trajectory between 90 degrees, the vertical pose, and 45 degrees in 2 seconds. This experiment replicates a pick and place operation, where the robot lifts, moves, and places an object between two points following a defined trajectory.

4.2.2. Experiment Type 2. The motor position $\left(\theta_{m}\right)$ tracks a 4-second sinusoidal trajectory between 90 degrees and 45 degrees. This experiment is used to prove the stability of the algorithm when a persistent input is applied. Identification stability is not evident taking into account that the algorithm is based on unstable filters.

4.2.3. Experiment Type 3. The motor is blocked at 0 degrees $\left(\theta_{m}=0\right)$, and then the link is gently hit in its tip. This experiment reproduces those cases where the controlled robot is not capable of completely cancelling the vibrations at the tip position or the robot at rest picks or releases an unknown payload.

Before ending this section, we present the motor position $\left(\theta_{m}\right)$ and torque $(\Gamma)$ measurements for each experiment type. Due to space constrains, we only display the measurements for the payload mass $m_{2}$. The plots for the payload masses $m_{1}$ and $m_{3}$ are similar and do not add value to the discussion. Figures 4, 5, and 6, show the sensor measurements used 


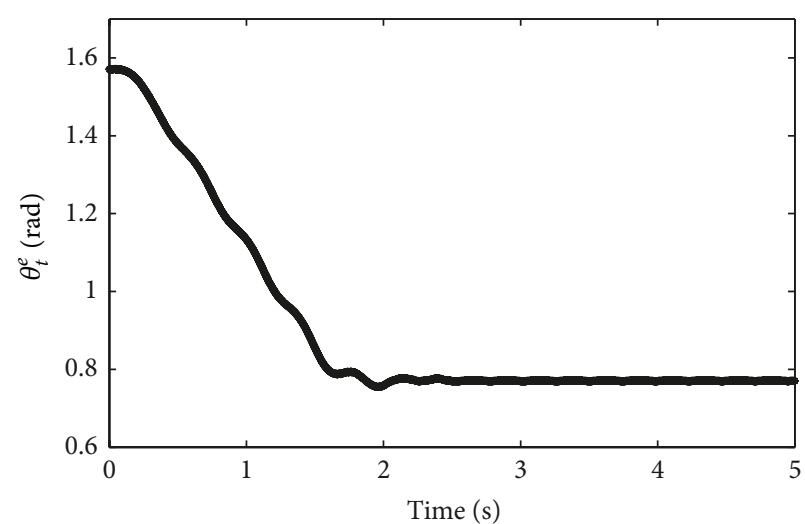

(a)

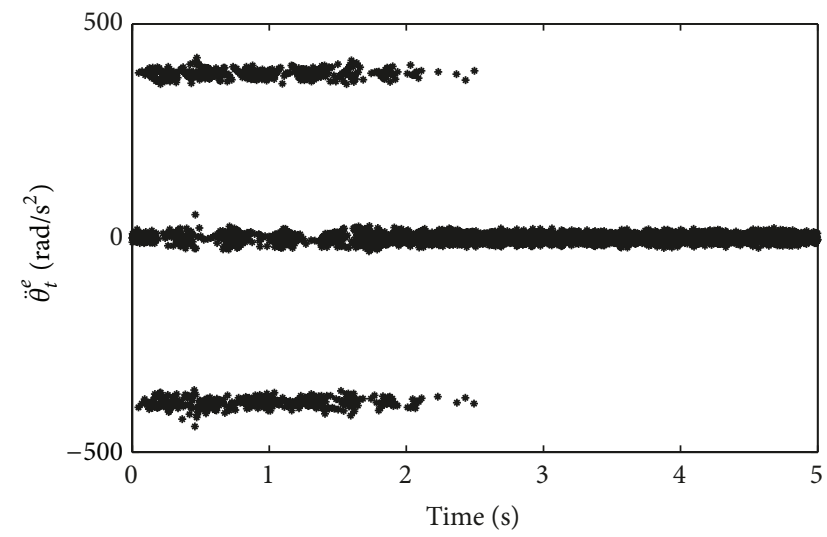

(c)

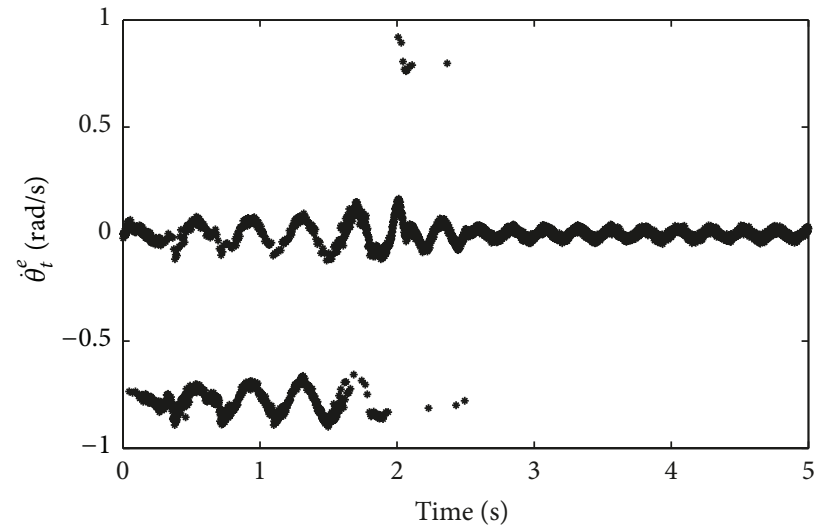

(b)

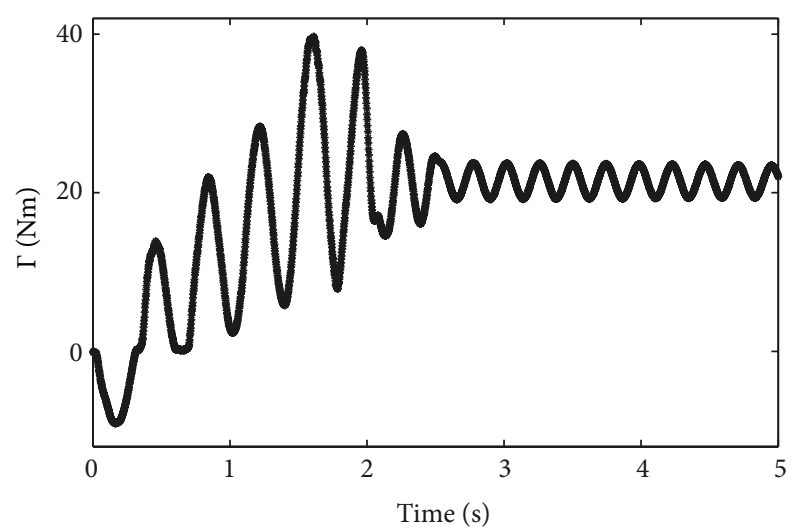

(d)

FIGURE 3: Example of the signals involved in the identification algorithm to discuss (a) estimation of the tip position $\left(\theta_{t}^{e}\right)$, (b) first-order numerical time differentiation of the estimation of the tip position $\left(\dot{\theta}_{t}^{e}\right)$, (c) second-order numerical time differentiation of the estimation of the tip position $\left(\ddot{\theta}_{t}^{e}\right)$, and $(\mathrm{d})$ coupling torque $(\Gamma)$.

TABLE 2: Algebraic identification: convergence time for the different experiments and payloads.

\begin{tabular}{|c|c|c|c|c|c|c|c|c|c|}
\hline \multirow{2}{*}{ Errors } & \multicolumn{3}{|c|}{ Experiment type 1} & \multicolumn{3}{|c|}{ Experiment type 2} & \multicolumn{3}{|c|}{ Experiment type 3} \\
\hline & $10 \%$ & $5 \%$ & $2 \%$ & $10 \%$ & $5 \%$ & $2 \%$ & $10 \%$ & $5 \%$ & $2 \%$ \\
\hline Payload $m_{1}$ & $0.57 \mathrm{~s}$ & $0.77 \mathrm{~s}$ & $1.08 \mathrm{~s}$ & $0.68 \mathrm{~s}$ & $0.84 \mathrm{~s}$ & $1.24 \mathrm{~s}$ & $0.022 \mathrm{~s}$ & $0.028 \mathrm{~s}$ & $0.030 \mathrm{~s}$ \\
\hline Payload $m_{2}$ & $0.33 \mathrm{~s}$ & $0.61 \mathrm{~s}$ & $1.27 \mathrm{~s}$ & $0.40 \mathrm{~s}$ & $0.65 \mathrm{~s}$ & $0.88 \mathrm{~s}$ & $0.036 \mathrm{~s}$ & $0.132 \mathrm{~s}$ & $0.238 \mathrm{~s}$ \\
\hline Payload $m_{3}$ & $0.29 \mathrm{~s}$ & $0.39 \mathrm{~s}$ & $0.58 \mathrm{~s}$ & $0.58 \mathrm{~s}$ & $0.73 \mathrm{~s}$ & $1.16 \mathrm{~s}$ & $0.048 \mathrm{~s}$ & $0.050 \mathrm{~s}$ & $0.196 \mathrm{~s}$ \\
\hline
\end{tabular}

for the identification in the experiment types 1,2 , and 3 , respectively.

\subsection{Algebraic Identification Results: Convergence Times and} Precision. In this section we present the experimental results of applying the first two stages of the algebraic identification algorithm that we detailed in Section 3. The third stage of this algorithm will be addressed in the next section.

The first two stages of the identification method can be summarised in three steps: first, to use the sensor measurements, $\theta_{m}$ and $\Gamma$, to estimate the tip position $\theta_{t}^{e}$ by means of (3); second, to compute the functions $q(t), \beta(t), \xi(t)$, and $\eta(t)$ from (10); and, third, to sample these functions to obtain the matrices $A[k]$ and $B[k]$, as shown in (15), and use them to finally compute the payload mass by applying (16). Table 2 summarizes the performance of the algebraic estimation algorithm in terms of speed of convergence and precision. In this table, we present the convergence times of the algorithm for the three types of experiments presented above, when the estimates reach and stay in the band of $\pm 10 \%, \pm 5 \%, \pm 2 \%$ of the real payload mass value. The following paragraphs are devoted to the discussion of these results in the context of pursuing the implementation of an adaptive control.

The convergence rates, shown in Table 2, demonstrate that algebraic identification produces accurate and fast 

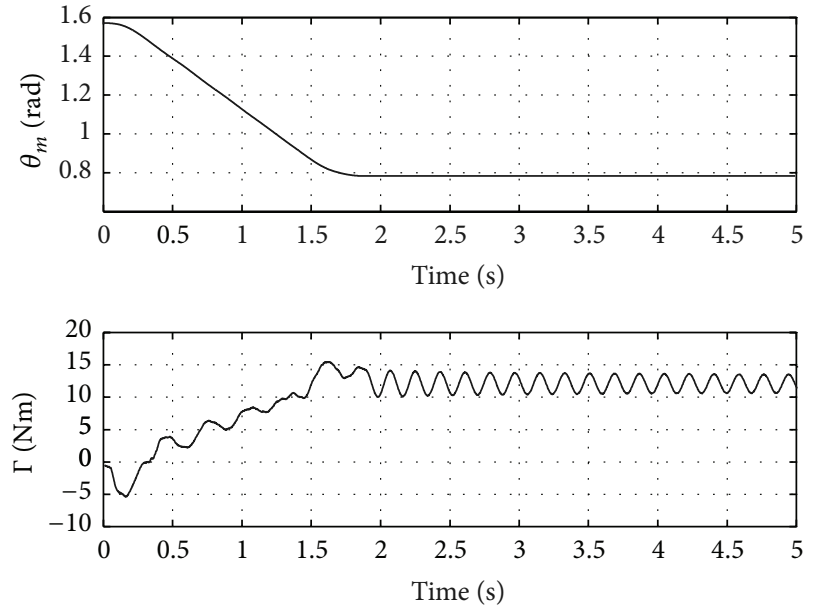

FIGURE 4: Experiment type 1: motor position and torque measurement for mass $m_{2}$.
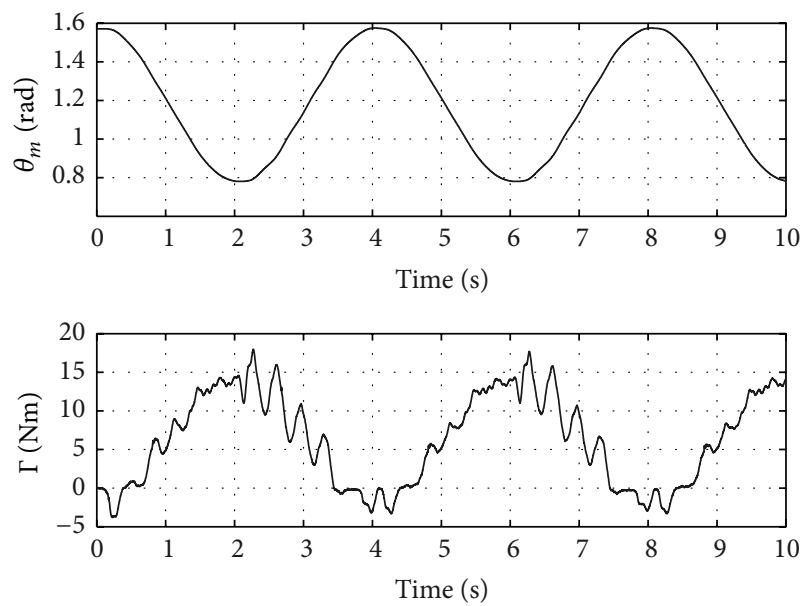

Figure 5: Experiment type 2: motor position and torque measurement for mass $m_{2}$.
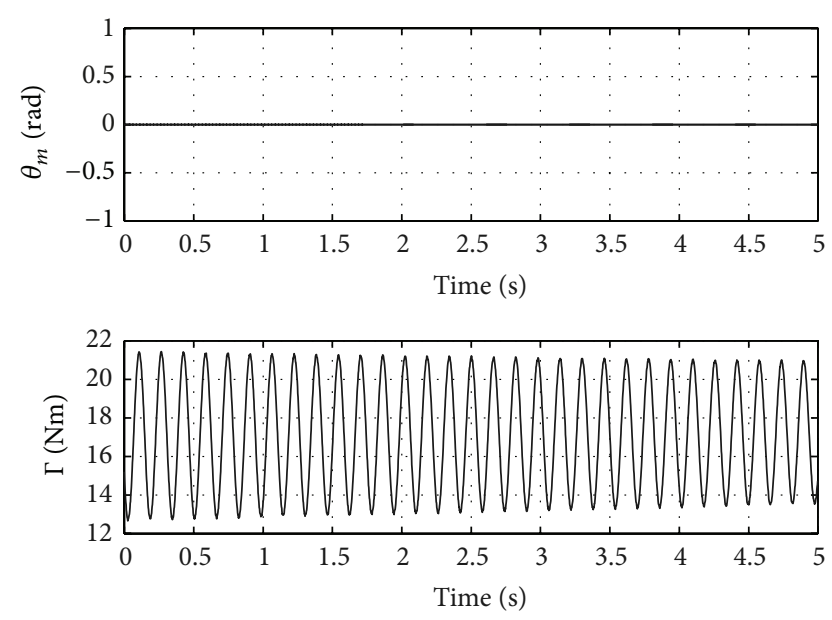

FIgURE 6: Experiment type 3: motor position and torque measurement for mass $m_{2}$. payload mass estimates in all the cases studied. This is in the experiments that considered large trajectories (experiment types 1 and 2), persistent inputs (experiment 2), and also in those that considered quasistatic situations (experiment type 3). Specifically speaking, this algorithm achieves payload estimates with errors lower than $5 \%$ in less than 0.84 seconds. This means that, in the specific case of the trajectory considered in the experiment type 1 , once the parameters in the adaptive controller have been updated, the controller has more than one half of the trajectory time interval to improve the tracking and the vibration suppression.

There are important differences in the convergence time between those experiments that involved large trajectories (experiment types 1 and 2) and the one that considered a quasistatic situation (experiment type 3). In the experiments of the first group, the payload estimates were achieved in an average time of $0.48,0.67$, and 1.03 seconds with errors of $10 \%$, $5 \%$, and $2 \%$, respectively, and with little difference between them. In the quasistatic situation, experiment type 3 , the algorithm needed $0.035,0.07$, and 0.15 seconds to guarantee the same margin errors. This means that in the experiment type 3 the payload estimation was achieved about 8 times faster than in the experiment types 1 and 2 . These differences should be taken into account by the adaptive controller.

4.4. Algebraic Identification Results: Resetting Algorithm. The purpose of the convergence criterium (18) is to automatically detect when the algebraic identification algorithm has converged in order to immediately reset the identification algorithm if necessary. In this section we present the results of this convergence criterium when it was applied to experiments type 1.

At this point, we assumed that the sensor measurements in the experiments type $1, \theta_{m}$ and $\Gamma$, were used to estimate the tip position $\theta_{t}^{e}$, as shown in (3), and, subsequently, to estimate the payload mass by applying (10), (15), and (16). The convergence criterium used the payload mass estimates provided by the previous algorithm and it was configured in terms of the tolerance parameter $(\Delta)$ and the windows size $(M)$. In the context of experiments type 1 , we assumed that it was desirable to have an identification algorithm capable of performing payload estimates in a period of time shorter than one half of the trajectory, that is, in less than $1 \mathrm{~s}$. With this condition in mind, we defined the tolerance parameter $(\Delta)$ of 0.015 and a windows size $(M)$ of 150 samples $(0.3 \mathrm{~s})$. Table 3 summarizes the performance of the resetting algorithm evaluated for these experiments. As can be seen in this table, convergence times stay less than or equal to 0.9 seconds for all the payload masses considered. It means that the payload mass is computed in the $45 \%$ of the time required for the trajectory. In the same table, it can be also noticed that the estimation errors are below the 5\% margin of error, which is an acceptable error for a potential adaptive control application. To count with a resetting algorithm is especially important because the convergence time varies depending on the type experiment trajectory performed. Taking estimates of the parameter based on a fixed time implies not exploiting the full potential of the identifier in terms of updating speed and, what it worse, it could make us take incorrect estimates 
TABLE 3: Algebraic identifier resetting algorithm: evaluation for experiment type 1 .

\begin{tabular}{lccc}
\hline & $\begin{array}{c}\text { Convergence } \\
\text { time }\end{array}$ & $\begin{array}{c}\text { Experiment type 1 } \\
\text { \% of the trajectory } \\
\text { duration }\end{array}$ & Error \\
\hline Payload $m_{1}$ & $0.90 \mathrm{~s}$ & $45.0 \%$ & $3.99 \%$ \\
Payload $m_{2}$ & $0.63 \mathrm{~s}$ & $31.7 \%$ & $4.61 \%$ \\
Payload $m_{3}$ & $0.65 \mathrm{~s}$ & $32.4 \%$ & $1.06 \%$ \\
\hline
\end{tabular}

of the parameter if the algorithm has not already converged. This is apparent in our experiment where, as it was pointed in the last section, the convergence time for the experiment type 3 was achieved in only a fraction of time required for the experiment types 1 and 2 .

4.5. Comparison with Least Square Fitting. In this section we compare the performance of the linear least squares fitting approach against the algebraic identification approach. For the purpose of a fair comparison between these, we considered two versions of the linear least squares fitting.

In the first case, we obtained the tip payload mass $(m)$ and viscous friction coefficient $(\nu)$ by applying the regular linear least square fitting to (1) that is linear in parameters. Here we considered the motor position $\left(\theta_{m}\right)$ and coupling torque $(\Gamma)$ measurements to compute an estimation of the tip position $\left(\theta_{t}^{e}\right)$ by taking into account expression (3). Taking these equations into account, the procedure is reduced to apply a linear least squares fitting to the following equation:

$$
\Gamma(t)=m\left(l^{2} \ddot{\theta}_{t}^{e}(t)+g l \cos \left(\theta_{t}^{e}(t)\right)\right)+v\left(\dot{\theta}_{t}^{e}(t)\right),
$$

where the first- and second-order time derivatives of the estimation of the tip position, $\left(\dot{\theta}_{t}^{e}\right)$ and $\left(\ddot{\theta}_{t}^{e}\right)$, respectively, were computed by numerical differentiation.

In the second case, we filtered the terms of (19) through a low-pass second-order Butterworth filter, before applying the regular linear least square fitting indicated in the first case. This is shown in

$$
\begin{aligned}
F\{\Gamma(t)\}= & m F\left\{l^{2} \ddot{\theta}_{t}^{e}(t)+g l \cos \left(\theta_{t}^{e}(t)\right)\right\} \\
& +\nu F\left\{\dot{\theta}_{t}^{e}(t)\right\},
\end{aligned}
$$

where the letter $F$ indicates the low-pass filtering operation. This filtering operation was performed to soften the effects of the noise present in the input signals of the linear squares fitting. The noise effects were shown in Figure 3.

Looking at the convergence rates of the regular least squares fitting, shown in Table 4, it is easy to evidence that this method is far behind in performance in comparison to the algebraic identifier. If we consider all the experiment types, this method requires 3.27 seconds to reach the $10 \%$ margin error while the algebraic identification algorithm achieves this in only 0.68 seconds. This means that the algebraic identifier is about 5 times faster than the regular least squares fitting.

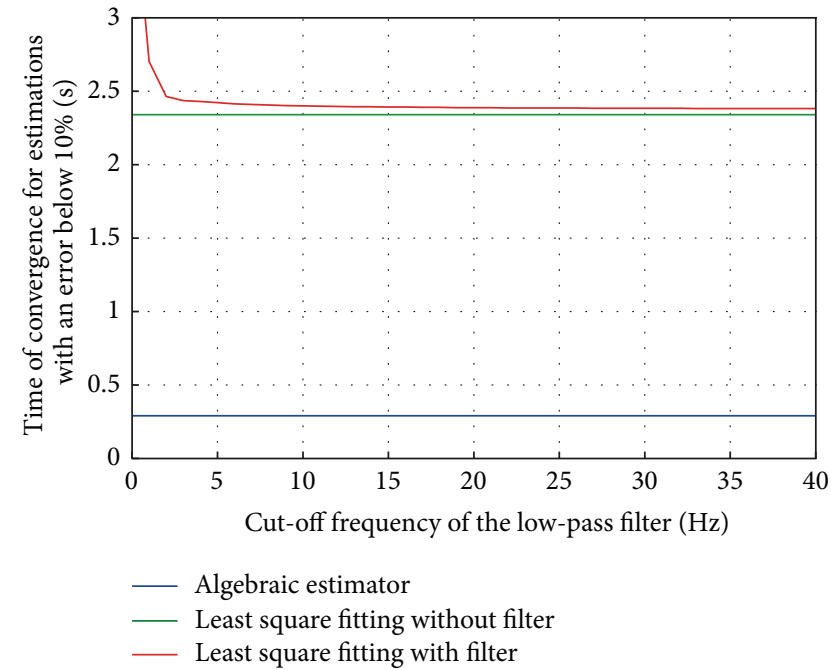

FIGURE 7: Comparison of the convergence times respect cutoff frequency for the identification of the payload mass $m_{3}$ in experiment type 1 .

On the other hand, the performance of the filtered version of the linear least square algorithm did not show any significant and consistent improvement in comparison with its nonfiltered version or the algebraic identification algorithm. In this performance study, cut-off frequencies between 0 and $40 \mathrm{~Hz}$ were considered in filter $F$ of (20) to deal with the noise present in the measurements. Discretizing the filter using the bilinear transform and a sampling time of $T_{s}=2 \mathrm{~ms}$, the poles $P_{i}$ of the filter must fulfill Haykin's condition $\left|P_{i} T_{s}\right|<0.5$ [27]. This implies that the maximum cut-off frequency of the filter is $0.5 / 2 \pi T_{s}=39.7 \mathrm{~Hz} \approx$ $40 \mathrm{~Hz}$. To illustrate the performance of this identification method, Figure 7 shows its convergence time with respect to different cut-off frequencies. This graphic presents the results of identification of the payload mass $m_{3}$ in the experiment type 1 . However, similar results are obtained from the other experiment types and masses. For ease of comparison with the other methods, the convergence times for the nonfiltered linear least squares algorithm and the algebraic algorithm are also indicated in Figure 7.

Figures 8, 9, and 10 show comparisons of the identification algorithms here discussed. A cut-off frequency of $10 \mathrm{~Hz}$ was considered for the filtered version of the linear least squares algorithm.

\section{Conclusions}

In this paper, an online algebraic identification algorithm has been presented for the identification of the payload mass of a single-link flexible robot moving under gravity. This algorithm was designed for a specific nonlinear dynamic structure that can be arranged to be linear in parameters. The algorithm considers as inputs the motor position and the torque measured at the base of the link. Its performance has been experimentally evaluated in situations that involve large trajectories (experiment types 1 and 2), trajectories that 
TABLE 4: Linear least squares fitting: convergence time for the different experiments and payloads.

\begin{tabular}{|c|c|c|c|c|c|c|c|c|c|}
\hline \multirow{2}{*}{ Errors } & \multicolumn{3}{|c|}{ Experiment type 1} & \multicolumn{3}{|c|}{ Experiment type 2} & \multicolumn{3}{|c|}{ Experiment type 3} \\
\hline & $10 \%$ & $5 \%$ & $2 \%$ & $10 \%$ & $5 \%$ & $2 \%$ & $10 \%$ & $5 \%$ & $2 \%$ \\
\hline Payload $m_{1}$ & $2.30 \mathrm{~s}$ & $>5 \mathrm{~s}$ & $>5 \mathrm{~s}$ & $1.30 \mathrm{~s}$ & $1.45 \mathrm{~s}$ & $2.94 \mathrm{~s}$ & $1.92 \mathrm{~s}$ & $4.27 \mathrm{~s}$ & $>5 s$ \\
\hline Payload $m_{2}$ & $2.15 \mathrm{~s}$ & $>5 \mathrm{~s}$ & $>5 \mathrm{~s}$ & $1.30 \mathrm{~s}$ & $1.46 \mathrm{~s}$ & $>5 \mathrm{~s}$ & $3.27 \mathrm{~s}$ & $>5 \mathrm{~s}$ & $>5 \mathrm{~s}$ \\
\hline Payload $m_{3}$ & $2.34 \mathrm{~s}$ & $>5 \mathrm{~s}$ & $>5 \mathrm{~s}$ & $1.40 \mathrm{~s}$ & $1.42 \mathrm{~s}$ & $2.95 \mathrm{~s}$ & $0.49 \mathrm{~s}$ & $3.70 \mathrm{~s}$ & $>5$ \\
\hline
\end{tabular}

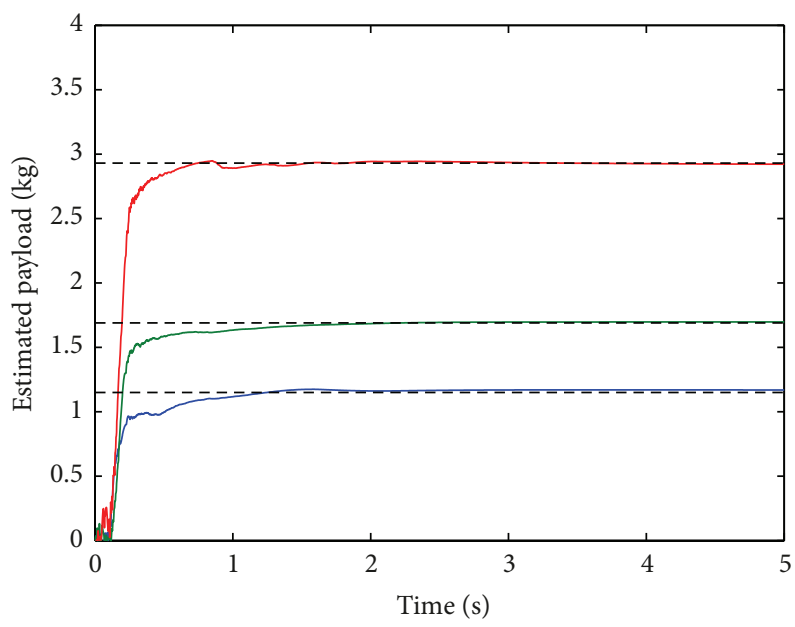

Estimation of the $1.15 \mathrm{~kg}$ payload

Estimation of the $1.69 \mathrm{~kg}$ payload

Estimation of the $2.93 \mathrm{~kg}$ payload

- - - Payload actual values

(a)

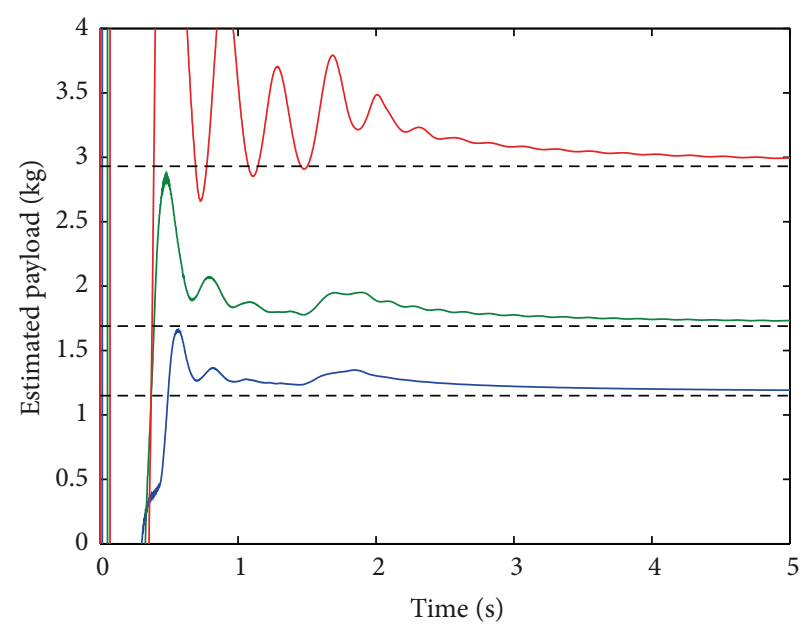

Estimation of the $1.15 \mathrm{~kg}$ payload

Estimation of the $1.69 \mathrm{~kg}$ payload

- Estimation of the $2.93 \mathrm{~kg}$ payload

- - - Payload actual values

(b)

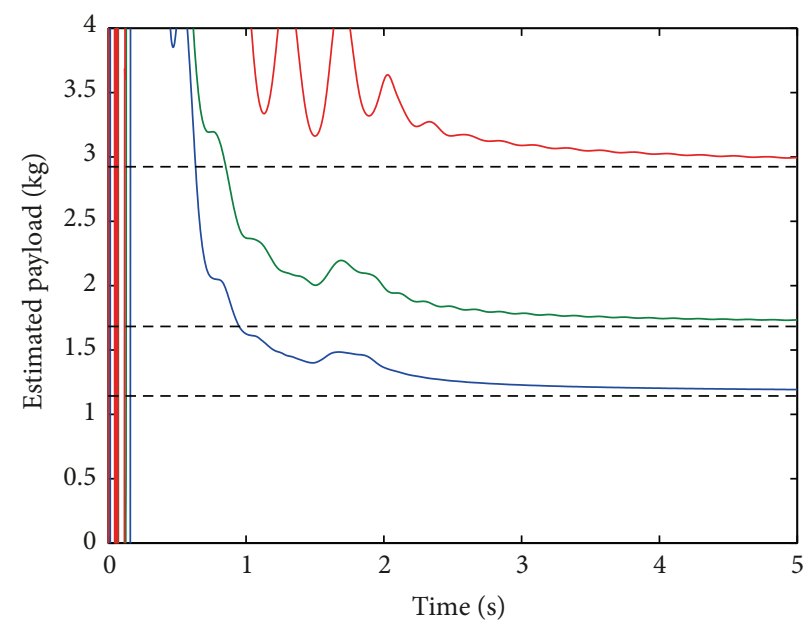

Estimation of the $1.15 \mathrm{~kg}$ payload
_ Estimation of the $1.69 \mathrm{~kg}$ payload
_- Estimation of the $2.93 \mathrm{~kg}$ payload
- Payload actual values

(c)

FIGURE 8: Experiment type 1: (a) algebraic identifier, (b) least square fitting, and (c) least square fitting with filter. 


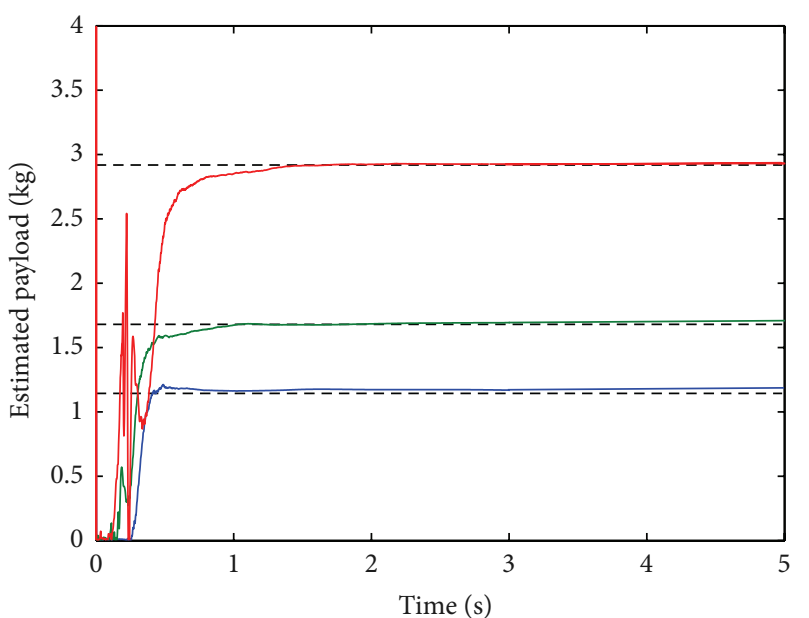

Estimation of the $1.15 \mathrm{~kg}$ payload

- Estimation of the $1.69 \mathrm{~kg}$ payload

_ Estimation of the $2.93 \mathrm{~kg}$ payload - - Payload actual values

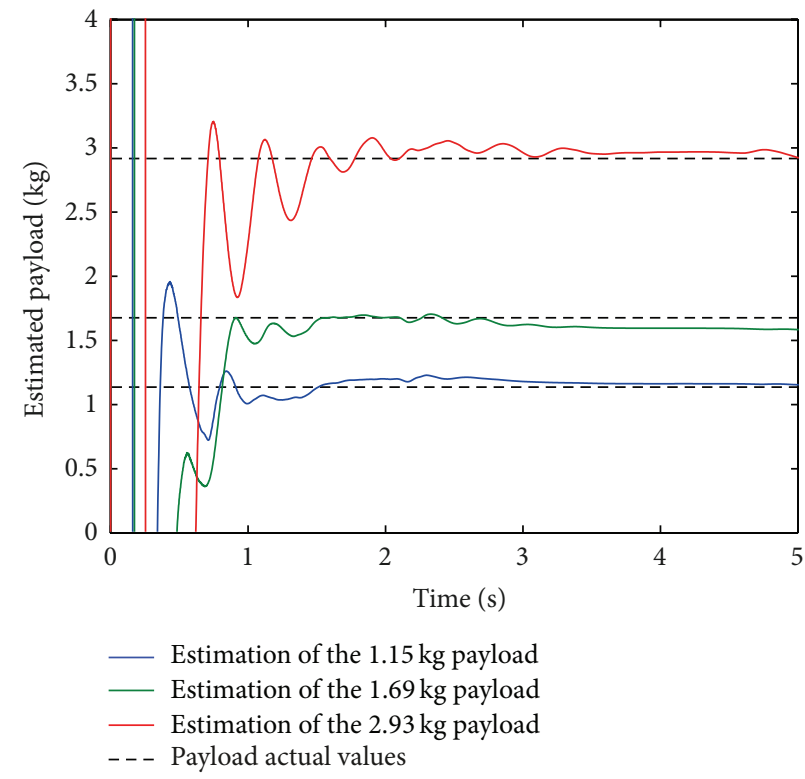

(b)

(a)
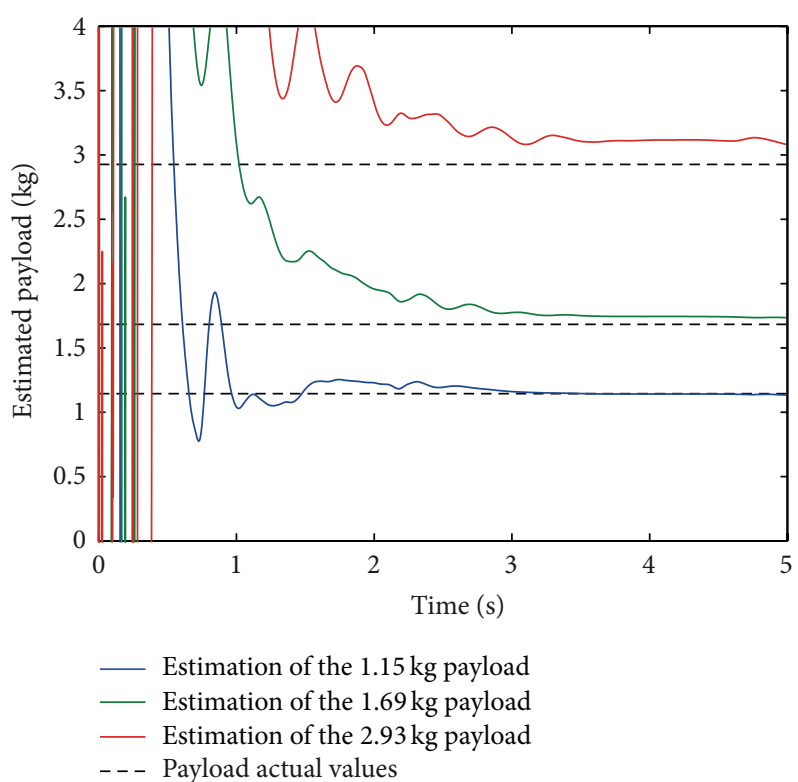

(c)

FIgURE 9: Experiment type 2: (a) algebraic identifier, (b) least square fitting, and (c) least square fitting with filter.

persistently excited the link (experiment type 2), and in a quasistatic situation (experiment type 3), where the flexible link, initially at rest, is externally perturbed. The performance analysis of this algorithm shows that it is capable of achieving estimates in less than 0.84 seconds with an error lower than $5 \%$. For large trajectories, similar to those we considered in the experiments (45 degrees in 2 seconds), this might be an acceptable convergence rate if it has to be included in an indirect adaptive control implementation. An adaptive controller that considers this identification algorithm might count with more than one half of the trajectory lasting time to improve the trajectory tracking and the vibration suppression.

To highlight the performance of our identification algorithm, we presented a comparison with a linear least square fitting. Other identification algorithms described in the scientific literature and referenced in Introduction have not been included in this comparison because they are not suited to deal with nonlinear dynamics. In particular, two least squares fitting based algorithms were considered: the regular least square fitting and a prefiltered version. For estimations with errors below $10 \%$, the results of 


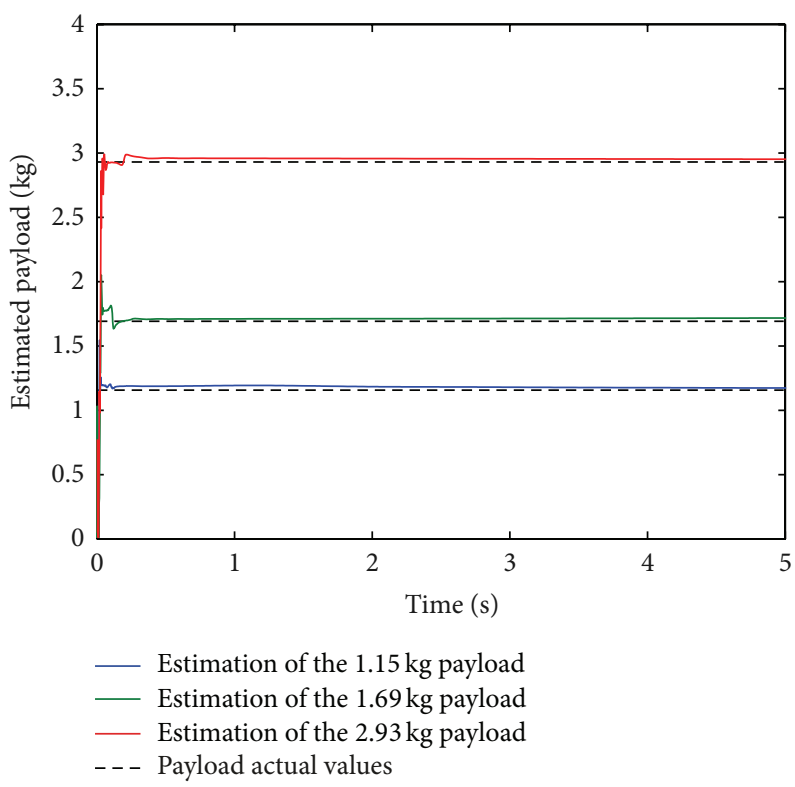

(a)

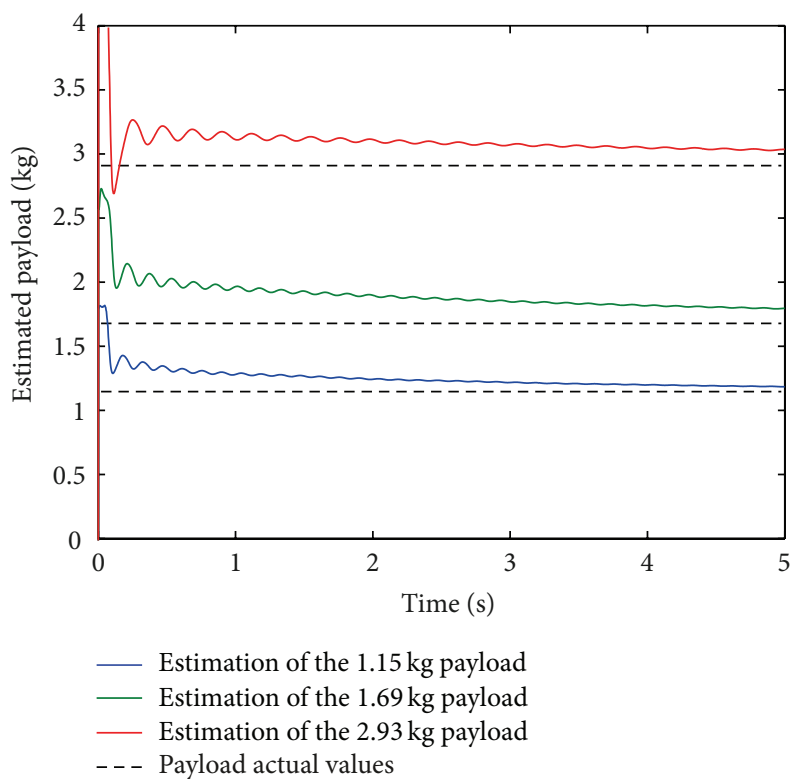

(b)

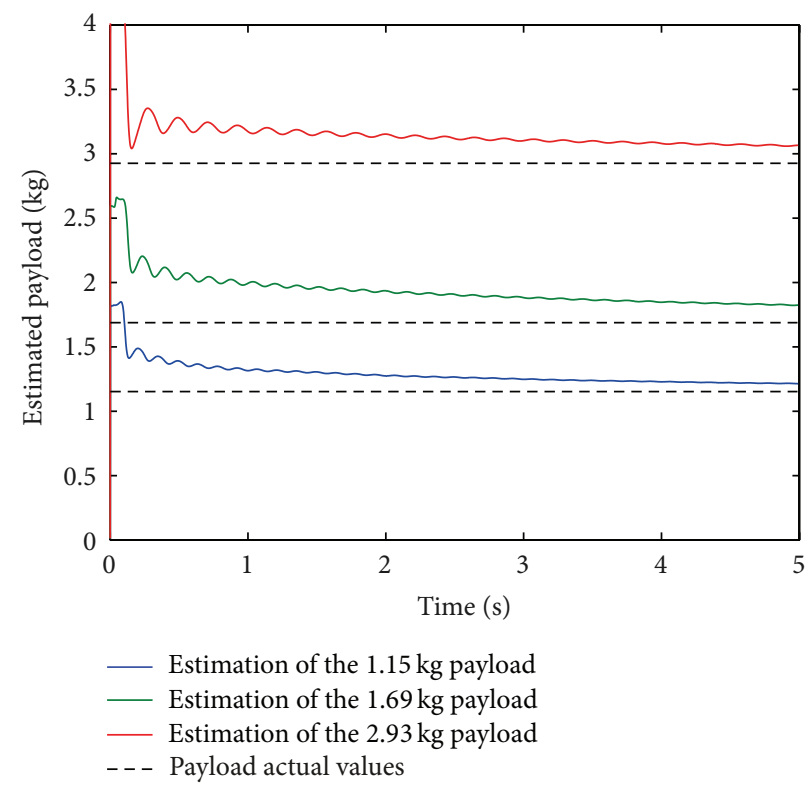

(c)

FIGURE 10: Experiment type 3: (a) algebraic identifier, (b) least square fitting, and (c) least square fitting with filter.

the comparison show that the algebraic identification algorithm is about 5 times faster than the regular least squares fitting. The filtered variant, for its part, did not show any improvement in terms of time of convergence in comparison with its regular version. The performance superiority of our algorithm over the linear least square fitting can be attributed, in good part, to the fact that the algebraic identifier does not require any time derivative of the measurements. This trait avoids the noise amplification problems that the other methods present and, moreover, allows using sensors of the link position with relatively low resolution, as we showed in our experiments.

In this work we implemented a resetting algorithm to detect when the estimation convergence is achieved. This algorithm was tested in some experiments that reproduce the typical trajectory tracking performed in a pick and place task (experiment type 1). Its performance demonstrates its feasibility for a potential adaptive control application. 
Our further work will be devoted to the study of adaptive control laws based on the estimation algorithm here developed.

\section{Conflict of Interests}

The authors declare that there is no conflict of interests regarding the publication of this paper.

\section{Acknowledgments}

This paper was sponsored by the Spanish FPU12/00984 Program (Ministerio de Educacion, Cultura y Deporte). It was also sponsored by the Spanish Government Research Program with the Project DPI2012-37062-CO2-01 (Ministerio de Economia y Competitividad) and by the European Social Fund.

\section{References}

[1] V. Feliu, "Robots flexibles: hacia una generación de robots con nuevas prestaciones," Revista Iberoamericana de Automática e Informática Industrial, vol. 3, no. 3, pp. 24-41, 2009.

[2] S. K. Dwivedy and P. Eberhard, "Dynamic analysis of flexible manipulators, a literature review," Mechanism and Machine Theory, vol. 41, no. 7, pp. 749-777, 2006.

[3] M. Benosman and G. Le Vey, "Control of flexible manipulators: a survey," Robotica, vol. 22, no. 5, pp. 533-545, 2004.

[4] B. Siciliano, B.-S. Yuan, and W. J. Book, "Model reference adaptive control of a link flexible arm," in Proceedings of the 25th IEEE Conference on Decision and Control, pp. 91-95, IEEE, Athens, Greece, December 1986.

[5] J. Yuh, "Application of discrete-time model reference adaptive control to a flexible single-link robot," Journal of Robotic Systems, vol. 4, no. 5, pp. 621-630, 1987.

[6] S. Yurkovich and A. P. Tzes, "Experiments in identification and control of flexible-link manipulators," IEEE Control Systems Magazine, vol. 10, no. 2, pp. 41-46, 1990.

[7] S. Bittanti, M. Campi, and S. M. Savaresi, "Unbiased estimation of a sinusoid in colored noise via adapted notch filters," Automatica, vol. 33, no. 2, pp. 209-215, 1997.

[8] G. Obregón-Pulido, B. Castillo-Toledo, and A. Loukianov, "A globally convergent estimator for n-frequencies," IEEE Transactions on Automatic Control, vol. 47, no. 5, pp. 857-863, 2002.

[9] J. R. Trapero, H. Sira-Ramírez, and V. F. Batlle, "An algebraic frequency estimator for a biased and noisy sinusoidal signal," Signal Processing, vol. 87, no. 6, pp. 1188-1201, 2007.

[10] D. M. Rovner and R. H. Cannon, "Experiments toward on-line identification and control of a very flexible one-link manipulator," The International Journal of Robotics Research, vol. 6, no. 4, pp. 3-19, 1987.

[11] T.-C. Yang, J. C. S. Yang, and P. Kudva, "Adaptive control of a single-link flexible manipulator with unknown load," IEE Proceedings D: Control Theory and Applications, vol. 138, no. 2, pp. 153-159, 1991.

[12] J.-S. Chen and C.-H. Menq, "Experiments on the payloadadaptation of a flexible one-link manipulation with unknown payload," in Proceedings of the IEEE International Conference on Robotics and Automation, pp. 1614-1619, IEEE, Cincinnati, Ohio, USA, May 1990.

[13] J. J. Feliu, V. Feliu, and C. Cerrada, "Load adaptive control of single-link flexible arms based on a new modeling technique,"
IEEE Transactions on Robotics and Automation, vol. 15, no. 5, pp. 793-804, 1999.

[14] G. Takács, T. Polóni, and B. Rohal'-Ilkiv, "Adaptive model predictive vibration control of a cantilever beam with real-time parameter estimation," Shock and Vibration, vol. 2014, Article ID 741765, 15 pages, 2014

[15] J. Becedas, J. R. Trapero, V. Feliu, and H. Sira-Ramírez, "Adaptive controller for single-link flexible manipulators based on algebraic identification and generalized proportional integral control," IEEE Transactions on Systems, Man, and Cybernetics, Part B: Cybernetics, vol. 39, no. 3, pp. 735-751, 2009.

[16] M. Fliess and H. Sira-Ramrez, "An algebraic framework for linear identification," ESAIM Control Optimisation and Calculus of Variations, vol. 9, pp. 151-168, 2003.

[17] A. San-Millan, J. C. Cambera, and V. Feliu, "Online algebraic identification of the payload changes in a single-link flexible manipulator moving under gravity," in Proceedings of the 19th IFAC World Congress, vol. 19, pp. 8397-8402, Cape Town, South Africa, 2014.

[18] H. Sira-Ramírez, C. García-Rodríguez, J. Cortés-Romero, and A. Luviano-Juárez, Algebraic Identification and Estimation Methods in Feedback Control Systems, John Wiley \& Sons, Chichester, UK, 2014.

[19] V. Feliu, K. S. Rattar, and H. B. Brown Jr., "Modeling and control of single-link flexible arms with lumped masses," Transactions of the ASME-Journal of Dynamic Systems, Measurement and Control, vol. 114, no. 1, pp. 59-69, 1992.

[20] J. R. Trapero, H. Sira-Ramírez, and V. F. Batlle, "On the algebraic identification of the frequencies, amplitudes and phases of two sinusoidal signals from their noisy sum," International Journal of Control, vol. 81, no. 3, pp. 505-516, 2008.

[21] C. Garcia-Rodriguez, J. A. Cortes-Romero, and H. Sira-Ramirez, "Algebraic identification and discontinuous control for trajectory tracking in a perturbed 1-DOF suspension system," IEEE Transactions on Industrial Electronics, vol. 56, no. 9, pp. 3665-3674, 2009.

[22] J. Cortés-Romero, C. García-Rodríguez, A. Luviano-Juárez, and H. Sira-Ramírez, "Algebraic parameter identification for induction motors," in Proceedings of the 37th Annual Conference of the IEEE Industrial Electronics Society (IECON’11), pp. 1734-1740, November 2011.

[23] A. San-Millan and V. Feliu, "A fast online estimator of the two main vibration modes of flexible structures from biased and noisy measurements," IEEE/ASME Transactions on Mechatronics, vol. 20, no. 1, pp. 93-104, 2015.

[24] R. Garrido and A. Concha, "An algebraic recursive method for parameter identification of a servo model," IEEE/ASME Transactions on Mechatronics, vol. 18, no. 5, pp. 1572-1580, 2013.

[25] E. Pereira, J. R. Trapero, I. M. Díaz, and V. Feliu-Batlle, "Algebraic identification of the first two natural frequencies of flexible-beam-like structures," Mechanical Systems and Signal Processing, vol. 25, no. 7, pp. 2324-2335, 2011.

[26] J. C. Cambera, J. A. Chocoteco, and V. Feliu, "Feedback linearizing controller for a flexible single-link arm under gravity and joint friction," in ROBOT2013: 1st Iberian Robotics Conference: Advances in Robotics, Vol. 2, vol. 253 of Advances in Intelligent Systems and Computing, pp. 169-184, Springer, Berlin, Germany, 2014.

[27] S. Haykin, "Unified treatment of recursive digital filtering," IEEE Transactions on Automatic Control, vol. 17, no. 1, pp. 113116, 1972. 

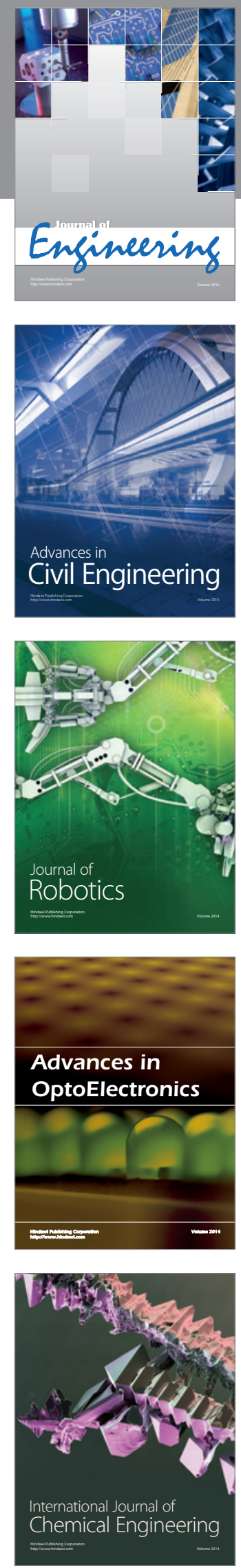

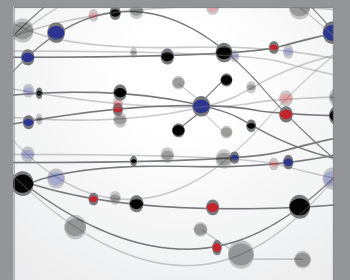

The Scientific World Journal
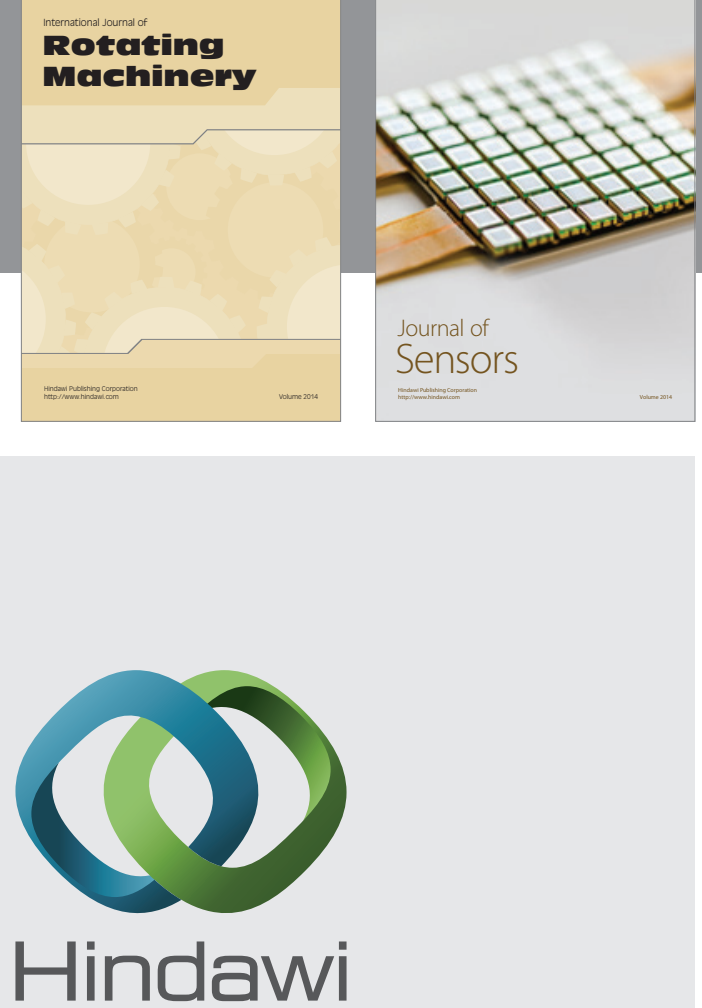

Submit your manuscripts at http://www.hindawi.com
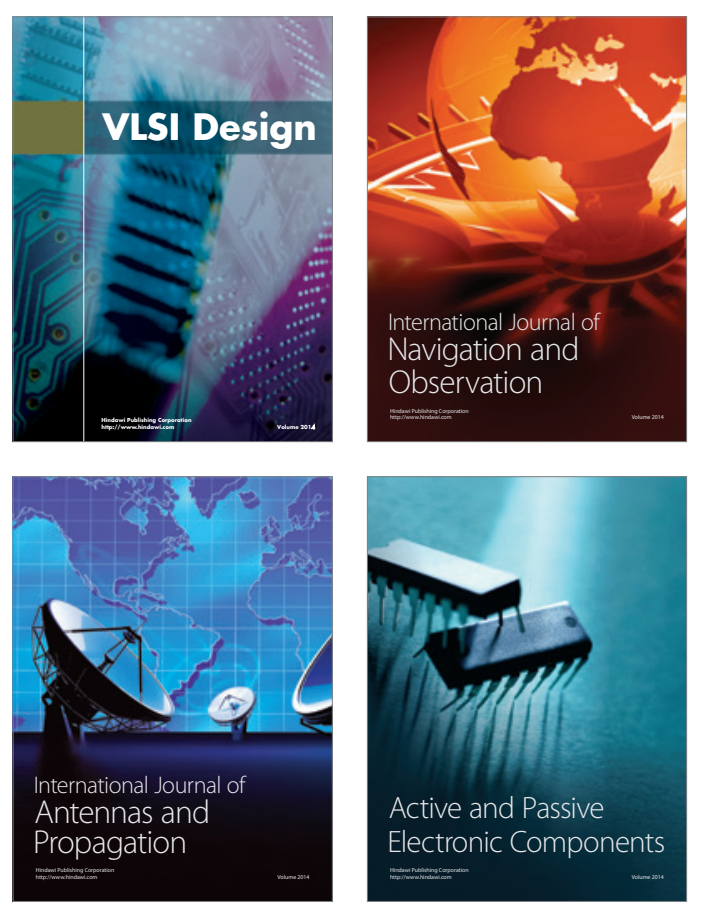
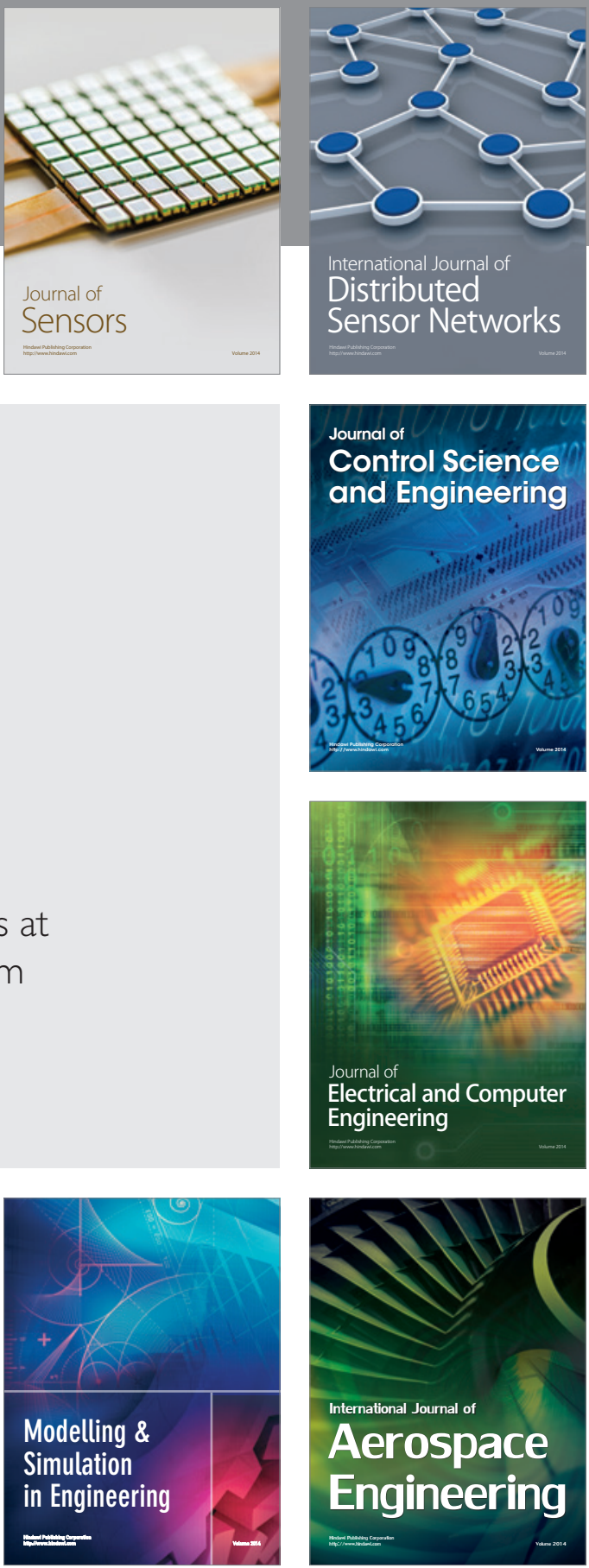

Journal of

Control Science

and Engineering
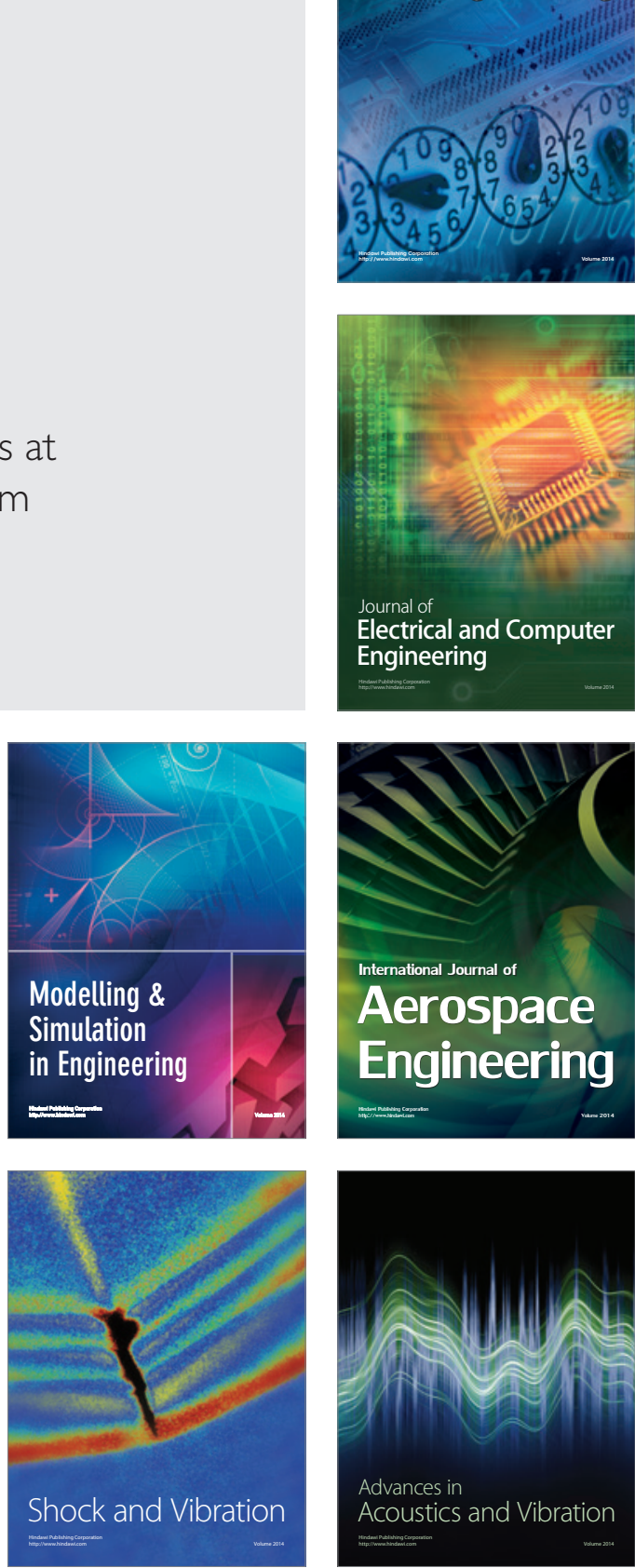